\title{
Effectiveness of medical treatment in overcoming the ureteral double-J stent related symptoms
}

\author{
Ugur Kuyumcuoglu, MD; Bilal Eryildirim, MD; Murat Tuncer, MD; Gokhan Faydaci, MD; Fatih Tarhan, MD; \\ Aydin Ozgül, MD
}

Dr. Lüffi Kirdar Training and Research Hospital 1. Urology Clinic, Istanbul, Turkey

Cite as: Can Urol Assoc J 2012;6:E234-E237. http://dx.doi.org/10.5489/cuaj.10143. Epub 2011 Sept 8 .

\section{Abstract}

Background: We investigated whether the frequency of lower urinary tract symptoms (LUTS) increased in patients in whom double-J stents were applied. We also evaluated several medical therapy protocols to treat symptoms related with ureteral stents.

Materials and Methods: A total of 108 patients, in whom unilateral double-j stent was applied during ureteral stone treatment, were included. Before the double-J stent was applied, all patients completed storage components of the "International Prostate Symptom Score" (IPSSs), quality of life components of the IPSS (IPSS-QOL) and "Overactive Bladder Questionnaire" (OABq) forms and scores were calculated. After the procedure, cases were randomized into 5 groups, an antiinflammatory was given to Group 1, spasmolytic to Group 2, anticholinergic to Group 3 and $\alpha$-blocker to Group 4. No additional drug was given to Group 5 as this control group. During the fourth week of the procedure, IPSSs, IPSS-QOL and $\mathrm{OABq}$ forms were again completed and scores were compared with the previous ones.

Results: When all the cases were evaluated, the IPSSs, IPSS-QOL and OABq scores of patients in whom the double-J stent was applied were statistically significantly higher the procedure. Compared to the control group, the cases where the double-J stent was applied showed a higher IPSSs, IPSS-QOL and OABq scores and none of the medical therapies could prevent this increase.

Interpretation: The frequency of LUTS increased in cases where the ureteral stent was applied and discomfort continued as long as the stent stayed in the body.

\section{Introduction}

Cystoscopic intraluminal placement of ureteral stents has become a routine practice in urology. Ureteral stents preserve urine flow from the kidney to the bladder in cases of ureteral obstruction (intrinsic or extrincis). In patients with obvious ureteral obstruction, the placement of a ureteral stent will restart urine transport and protect the kidney from possible risks. The stents also prevent urine extravasation after an operation or trauma.

One of the most preferred stents is the double-J stent with J-shaped ends on both sides. Ureteral double-j stents are generally placed for a temporary period. The duration of the stent may differ due to the reason of the application and the experience of the doctor. Although double-J stents are generally safe and well-tolerated, they have complications, such as pain, discomfort, urinary infection, recurrent obstruction, stent encrustation, stent fragmantation and stent migration. Their negative effects on quality of life and sexual functions have also been emphasized by some researchers. ${ }^{1,2}$ Research is ongoing in developing and validating questionnaires on symptoms related to ureteral stents and the effects of these symptoms on daily life.

A way to reduce symptoms related with stents might be to alter the design and material of the stents. Researchers continue to study the effect of several medical therapies on reducing the negative symptoms which affect quality of life. ${ }^{1,3}$

In this study, we investigated whether the frequency of lower urinary tract symptoms (LUTS) increased in patient in whom the double-J stents were applied. We also evaluated the significance of several medical therapies to treat the symptoms related with ureteral stents by using storage components of the "International Prostate Symptom Score" (IPSSs), quality of life components of the IPSS (IPSS-QOL) and "Overactive Bladder Questionnaire" (OABq) forms.

\section{Methods}

A total of 108 pateints (55 male, 53 female) were included in the study. Every patient had a unilateral double-J stent applied during ureteral stone treatment. The double-J stent was placed because of hydronephrosis prior to ESWL in 71 patients, where the stent was applied after lithotripsy with ureterorenoscopy in the remaining 37 patients.

Cases with lower urinary system infection, urogenital system tumour, history of previous urogenital system sur- 
gery, neurogenic bladder, uretral obstruction, bladder stone, overactive bladder, chronic prostatitis and benign prostatic hyperplasia were excluded. Approval was obtained by our Hospital Ethics Committee and all patients were informed about the study and gave their informed consent prior to their inclusion in the study.

Detailed anamnesis and physical examinations were done with all patients during their initial visit. Urine samples were taken for whole urine analysis and urine culture. Urinary system was assessed by direct urinary system $\mathrm{X}$-ray imaging and urinary system ultrasonography. Before the double-J stent was applied, all patients completed the storage components of the "International Prostate Symptom Score" (IPSSs) (questions 2, 4 and 7), quality of life components of the IPSS (IPSS-QOL) and "Overactive Bladder Questionnaire" (OABq) forms and their scores were calculated. ${ }^{4,5}$

The double-J stent was placed in patients under local anesthesia and intravenous sedation (dormicum, $0.05 \mathrm{mg} /$ $\mathrm{kg}$ ). In all cases, a $4.7 \mathrm{~F}$ (in diameter) and, depending on the patient, a 26- or 28-cm long polyurethane stent was placed (Marflow AG, Zürich, Switzerland). After the procedure, a direct urinary system X-ray imaging was done to confirm the stent was properly applied.

After the procedure, patients were randomized into 5 groups; patients in 4 of these 5 groups were administered an additional week of antibiotherapy: an antiinflammatory drug was given to Group1 (diclofenac potassium, $50 \mathrm{mg}$, 3 times daily, when needed), spasmolytic drug to Group 2 (flavoxate HCL 200 mg, 3 times daily, when needed), anticholinergic drug to Group 3 (tolterodine-L-tartarate SR $4 \mathrm{mg}$, once a day), and $\alpha$-blocker drug to Group 4 (doksazosin XL 4 mg, once a day). No additional drug was given to Group 5 as this was the control group. During the fourth week of the procedure, the IPSSs, IPSS-QOL and OABq questionnaires were once again completed and scores were compared with the previous ones.

To evaluate the results of the study, the GraphPad prism 5.0 program (GraphPad Software, Inc., La Jolla, CA) was used for the statistical analysis. During the assessment of the data, Wilcoxon and paired t tests were used for quantitative analysis. The differences of scores among the five groups were compared using covariance analysis (ANCOVA) before and after the double-J stent application. The results were analyzed with a $95 \%$ confidence interval and a significance of $p<0.05$.

\section{Results}

The mean age of the patients in the study was $43.34 \pm 1.39$. Regarding sex, age, body mass index (BMI) and stone size, statistically significant differences did not exist among groups (Table 1). When all the cases were evaluated, the IPSSs, IPSS-QOL and OABq scores of the patients in whom the double-J stent was applied were statistically significantly higher after the procedure, compared to before. When groups were separately evaluated, the IPSSs, IPSS-QOL and $\mathrm{OABq}$ scores were again statistically significant higher in all groups. Furthermore, the differences in scores among the five groups were not statistically significant before and after the stent placement. When comparing the groups to the control group, the patients in whom the double-J stent was applied showed higher IPSSs, IPSS-QOL and OABq scores; none of the medical therapies (anti-inflammatory, spasmolytic, anticholinergic, $\alpha$-blocker) could prevent this increase. The IPSSs, IPSS-QOL and OABq values of all the groups obtained before and after the procedure are presented (Table 2).

\section{Discussion}

Ureteral stent applications have become routine in urology. However, the double-J stent placement might lead to some unwanted adverse effects and complications. Several side effects, such as urinary infection, irritative urinary symptoms and suprapubic pain related with long-term double-J stents, might also cause psychological problems (i.e., insomnia, anxiety and depression). All these issus negatively affect work performance, sexual life and quality of life.

The pathophysiology of the symptoms related with ureteral stents is not clearly understood. However, it has been suggested that irritative symptoms are a result of the irritation of neuronal-rich trigon mucosa and flank pain, related with reflux. ${ }^{3}$ There are many studies investigating the side effects, complications that result from ureteral stent applications and the effects of these on quality of life. Leibovici and colleagues suggested that use of double-J stents can lead to several

Table 1. Patient characteristics

\begin{tabular}{|c|c|c|c|c|c|c|}
\hline & $\begin{array}{l}\text { Antiinflammatory } \\
\qquad(\mathrm{n}=23)\end{array}$ & $\begin{array}{c}\text { Spasmolytic } \\
(n=22)\end{array}$ & $\begin{array}{l}\text { Anticholinergic } \\
\quad(n=21)\end{array}$ & $\begin{array}{l}\text { Alpha-blocker } \\
\quad(n=21)\end{array}$ & $\begin{array}{l}\text { Control } \\
(n=21)\end{array}$ & $p$ \\
\hline $\operatorname{Sex}(M / F)$ & $10 / 13$ & $11 / 11$ & $12 / 9$ & $15 / 6$ & $7 / 14$ & 0.236 \\
\hline Age (year) & $45.71 \pm 3.26$ & $41.15 \pm 2.40$ & $41.53 \pm 2.99$ & $45.21 \pm 3.05$ & $42.94 \pm 3.88$ & 0.769 \\
\hline BMI $\left(\mathrm{kg} / \mathrm{m}^{2}\right)$ & $24.71 \pm 2.18$ & $24.42 \pm 1.85$ & $24.18 \pm 2.15$ & $24.13 \pm 2.15$ & $24.68 \pm 2.72$ & 0.882 \\
\hline Stone size $\left(\mathrm{mm}^{2}\right)$ & $65.81 \pm 20.28$ & $66.20 \pm 21.17$ & $67.32 \pm 24.95$ & $66.00 \pm 22.91$ & $68.83 \pm 27.00$ & 0.994 \\
\hline
\end{tabular}




\begin{tabular}{|c|c|c|c|c|c|c|c|}
\hline & & $\begin{array}{l}\text { Antiinflammatory } \\
\text { ( } n=23)\end{array}$ & $\begin{array}{l}\text { Spasmolytic } \\
(n=22)\end{array}$ & $\begin{array}{l}\text { Anticholinergic } \\
\quad(n=21)\end{array}$ & $\begin{array}{l}\text { Alpha-blocker } \\
(\mathbf{n}=\mathbf{2 1})\end{array}$ & $\begin{array}{l}\text { Control } \\
(n=21)\end{array}$ & $\boldsymbol{p}^{\prime}$ \\
\hline \multirow{2}{*}{ IPSSs } & Before & $3.09 \pm 0.59$ & $2.95 \pm 0.70$ & $4.00 \pm 0.75$ & $3.55 \pm 0.63$ & $3.22 \pm 0.63$ & 0.702 \\
\hline & After & $6.66 \pm 0.82^{\dagger+}$ & $6.50 \pm 0.84^{\dagger}$ & $6.42 \pm 0.97^{\ddagger}$ & $5.90 \pm 0.69^{\ddagger}$ & $5.27 \pm 0.77^{\ddagger}$ & \\
\hline \multirow{2}{*}{ IPSS-QOL } & Before & $1.61 \pm 0.27$ & $1.65 \pm 0.42$ & $1.78 \pm 0.34$ & $1.36 \pm 0.24$ & $1.44 \pm 0.38$ & 0.968 \\
\hline & After & $2.81 \pm 0.41^{\ddagger}$ & $3.15 \pm 0.51^{\ddagger}$ & $3.26 \pm 0.40^{\ddagger}$ & $3.05 \pm 0.40^{\dagger \dagger}$ & $3.00 \pm 0.44^{\ddagger}$ & \\
\hline \multirow{2}{*}{$\mathrm{OABq}$} & Before & $8,81 \pm 1,35$ & $7,40 \pm 1,28$ & $9,47 \pm 1,17$ & $7,37 \pm 1,34$ & $7,16 \pm 1,57$ & 0.990 \\
\hline & After & $13,95 \pm 1,59^{\ddagger}$ & $13,15 \pm 1,82^{\dagger \dagger}$ & $13,84 \pm 1,70^{\ddagger}$ & $12,11 \pm 1,46^{\dagger \dagger}$ & $12,83 \pm 2,37^{\dagger \dagger}$ & \\
\hline
\end{tabular}

*Comparisons of differences before and after measurements among the groups by using ANCOVA test; $\uparrow \mathrm{p}<0.001$ compared with before procedure; $\uparrow+p<0.01$ compared with before procedure; $¥ p<0.05$ compared with before procedure. IPSSs: Storage components of IPSS; IPSS-QOL: Quality of life; components of IPSS; OABq: Overactive bladder questionnaire.

side effects and cause negative effects on quality of life. These authors found common symptoms such as urgency, frequency and dysuria (respectively, 55\%, 50\% and 40\%). ${ }^{6}$

In their study of 146 cases, Damiano and colleagues showed that discomfort due to stent $(37.6 \%)$ and irritative urinary symptoms $(18.8 \%)$ were the most common complications in cases with a ureteral stent. The most interesting point in this study was that the frequency and severity of the complications increased in accordance with the duration of the stent use. ${ }^{7}$ Joshi and colleagues found that irritative urinary symptoms, incontinence and hematuria were present in $78 \%$ of the patients in whom the double-J stent was applied. In more than $80 \%$ of these patients, pain related to the stent affect their daily lives, sexual dysfunction was seen in $38 \%$ and a deterioration in work performance was seen in $58 \% .{ }^{8}$ In addition, Joshi and colleagues described a new questionnaire (Ureteral Stent Symptom Questionnaire) in 2003 that evaluated the symptoms caused by ureteral stents and their effects on general health, work performance and sexual life. ${ }^{9}$

Some investigators have attempted to explain stent-related morbidity, considering the lengths of the stents. They reported that the length of stent and crossing the midline of the distal end were significantly associated with stent-related symptoms. ${ }^{10,11}$ However this issue is controversial; there are some studies reporting that length and diameter of the stent does not significantly affect a patient's stent-related symptoms and quality of life. ${ }^{12,13}$ Further prospective randomized studies are needed in this field. The stents were 26 to 28 $\mathrm{cm}$ in length in our study. Long stents may be related with increased patient complaints and not comparing the different length of stents can be accepted as a limitation of our study. However, we believe that selection of the proper length of double-J stent may be an important factor in minimizing stent-related symptoms.

The effectiveness of several medical protocols on symptom improvement is also under investigation. Many medical therapies have been tested, such as analgesics, anticholinergics and $\alpha$-blockers, to deal with the irritative symptoms and pain related with ureteral stents. Deliveliotis and colleague showed that an $\alpha$-blocker, alfuzosin, might reduce symptoms, such as pain, frequency and urgency, related with double-J stents and may also decrease the need for analgesics. ${ }^{1}$ Beddingfield and colleagues, in a prospective, randomized and placebo-controlled study, revealed that alfuzosin lessened the complaints in patients in whom the ureteral stent was used. However, any significant decrease in narcotic drug use compared to control group was not observed in these cases. ${ }^{14}$ Norris and colleagues compared oxybutinin and phenazopyridine to treat stent-related symptoms and could not show any superiority of any of drug to overcome symptoms either compared to each other or to the control group. ${ }^{15} \mathrm{All}$ these treatments were not specific to symptoms, they rather cause a general improvement in symptoms, but may cause some other side effects as well. ${ }^{3}$

In this study, we investigated the side effects seen in patients in whom the double-J stent was applied and the effectiveness of various medical therapies to overcome these side effects. To evaluate LUTS more objectively, we used IPSSs, IPSS-QOL and OABq questionnaires. In all the cases in whom the double-j stent was applied, when the scores obtained before the procedure were compared with the ones obtained after the procedure, IPSSs, IPSS-QOL and $\mathrm{OABq}$ scores showed an increase and none of the medical therapy protocols we used (antiinflammatory, spasmolytic, anticholinergic, $\alpha$-blocker) showed a statistically significant superiority compared to each other and control group.

In our opinion, there is need for further studies and developments on new treatment strategies specific to LUTS related to stent placement. One other way of reducing stent-related symptoms might be to alter the design and material of the stents. Technology will lead the way in this field. However, the ideal biomaterial has yet to be discovered. With ongoing research in this area, further advances in ureteral stent design will continue to improve outcomes for patients who require stents. ${ }^{16,17}$

\section{Conclusion}

The frequency of LUTS increased in patients in whom the double-J stent was applied, with an increase in the IPSSs, IPSS-QOL and OABq scores. None of the medical therapies 
showed superiority compared to each other or to the control group in overcoming the symptoms that occur due to stents. In other words, discomfort continues as long as the stent stays in the body. To reduce these negative effects, the development of new treatment strategies new ways to improve the stent materials and designs are required.

Competıng interests: None declared.

Acknowledgment: We would like to thank Associate Professor Dr. Necdet Süt for his statistical analysis.

This paper has been peer-reviewed.

\section{References}

1. Deliveliotis C, Chrisofos M, Gougousis $E$, et al. Is there a role for alphal-blockers in treating double-J stentrelated symptoms? Urology 2006;67:35-9.

2. Sighinolfi MC, Micali S, De Stefani $S$ et al. Indwelling ureteral stents and sexual health: a prospective, multivariate analysis. J Urol 2007;178:229-31.

3. Duvdevani $M$, Chew BH, Denstedt JD. Minimizing symptoms in patients with ureteric stents. Curr Opin Urol 2006; 16:77-82.

4. Barry MJ, Fowler Jr FJ, O'Leary MP, et al. The American Urological Association Symptom Index for benign prostatic hyperplasia. J Urol 1992;148:1549-57.
5. Coyne K, Revicki D, Hunt $T$, et al. Psychometric validation of an overactive bladder symptom and healthrelated quality of life questionnaire: the OAB-q. Qual Life Res 2002;11:563-74.

6. Leibovici D, Cooper A, Lindner A, et al. Ureteral stents: morbidity and impact on quality of life. Isr Med Assoc J 2005;7:491-4.

7. Damiano R, Oliva A, Esposito $\mathrm{T}$, et al. Early and late complications of double pigtail ureteral stent. Urol Int 2002;69:136-40.

8. Joshi HB, Stainthorpe A, MacDonagh RP, et al. Indwelling ureteral stents: evaluation of symptoms, quality of life and utility. J Urol 2003;169:1065-9.

9. Joshi HB, Newns N, Stainthorpe A, et al. Ureteral stent symptom questionnaire: development and validation of a multidimensional quality of life measure. I Urol 2003;169:1060-4.

10. Rane A, Saleemi A, Cahill D, et al. Have stent-related symptoms anything to do with placement technique? J Endourol 2001;15:741-5.

11. Ho CH, Chen SC, Chung SD, et al. Determining the appropriate length of a double-pigtail ureteral stent by both stent configurations and related symptoms. J Endourol 2008;22:1427-31.

12. Damiano R, Autorino $R$, De Sio $M$, et al. Does the size of ureteral stent impact urinary symptoms and quality of life? A prospective randomized study. Eur Urol 2005;48:673-8.

13. Irani J, Siquier J, Pirès C, et al. Symptom characteristics and the development of tolerance with time in patients with indwelling double-pigtail ureteric stents. BJU Int 1999;84:276-9.

14. Beddingfield R, Pedro RN, Hinck $B$, et al. Alfuzosin to relieve ureteral stent discomfort: a prospective, randomized, placebo controlled study. J Urol 2009:181:170-6.

15. Norris RD, Sur RL, Springhart WP, et al. A prospective, randomized, double-blinded placebo-controlled comparison of extended release oxybutynin versus phenazopyridine for the management of postoperative ureteral stent discomfort. Urology 2008;71:792-5.

16. Duvdevani $M$, Chew BH, Denstedt JD. Minimizing symptoms in patients with ureteric stents. Curr Opin Urol 2006;16:77-82.

17. Beiko DT, Knudsen BE, Denstedt JD. Advances in ureteral stent design. J Endourol 2003; 17:195-9.

Correspondence: Dr. Bilal Eryildirim, Atalar Cad. Yakutlar Sitesi G/11 - Kartal, Istanbul, Turkey; fax:+90 216 3833193; bilaleryildirim@yahoo.com 